\title{
Incentivizing Firearm Safety and Storage Counseling in the US Health Care System: a Path Forward
}

\author{
Laura C. Prater, $P h D, M P H, M H A^{1,2}$, Alice Ellyson, $M S, P h D^{1,3}$, and Stephan D. Fihn, \\ $M D, M P H^{4,5}$
}

${ }^{1}$ Harborview Injury Prevention and Research Center, Harborview Medical Center, University of Washington, 401 Broadway Suite 2075, Seattle, WA, USA; ${ }^{2}$ Department of Epidemiology, School of Public Health, University of Washington, Seattle, WA, USA; ${ }^{3}$ Department of Pediatrics, University of Washington, Seattle, WA, USA; ${ }^{4}$ Department of Medicine, University of Washington, Seattle, WA, USA; ${ }^{5}$ Department of Health Services, School of Public Health, University of Washington, Seattle, WA, USA.

J Gen Intern Med 36(1 1):3568-70

DOI: $10.1007 / \mathrm{s} 11606-021-06800-\mathrm{x}$

(c) Society of General Internal Medicine 2021

$\mathrm{W}$ hile social isolation and perceptions of hopelessness during the COVID-19 pandemic have raised alarm about increasing suicide rates, the epidemic of suicide using firearms long preceded our current predicament. Firearms contributed to the deaths of nearly 40,000 people in 2018 , with two-thirds of those deaths attributable to suicide. Suicide is a leading cause of death for both adolescents and middleaged adults. Among Americans over age 65, firearms are the most common method used in suicide, particularly among men. ${ }^{1}$ Firearms are also the most lethal method of self-harm, with $90 \%$ of suicide attempts resulting in death. ${ }^{2}$

The number of firearms in homes increased in 2020, driven by fear of interpersonal violence, with many stored unsafely. ${ }^{3}$ Appropriately restricting access to those at high-risk and promoting safe firearm storage are currently the most feasible public health approaches to prevent suicide. Despite complexities in the delivery and financing of the US health care system, we believe these strategies to help prevent suicides involving firearms can be promoted through existing mechanisms.

Nearly half of those who die by suicide had an encounter with a health care professional in an inpatient, outpatient, or emergency room visit within four weeks of their death and up to $80 \%$ in the year prior. ${ }^{4}$ Assessments by clinicians of patient's access to lethal means, such as firearms, have been linked to a reduction in suicide rates in a large sample of high-risk adults. ${ }^{5}$ Accordingly, more than 45 medical professional societies have adopted guidance recommending that physicians counsel patients on firearm safety when clinically relevant. ${ }^{6}$ Nevertheless, while some health care providers regularly counsel patients about firearm safety, the majority do not.

Received January 26, 2021

Accepted April 1, 2021

Published online April 26, 2021
Three key barriers exist on the path toward meaningful integration of firearm safety and storage planning into clinical practice: (1) competing priorities during the clinical encounter, (2) lack of system-level prioritization of firearms safety and storage planning, and (3) the need for more rigorous evidence to understand the impact of firearm safety and storage counseling. We discuss recommendations for proceeding despite these barriers, through the integration of screening into routine clinical encounters, as well as potential tools for encouraging the delivery of firearm safety and storage counseling.

A suitable path forward is one that promotes firearm safety and storage planning through existing mechanisms in the US health care system. For veterans, the US Preventive Services Task Force (USPSTF) recommends screening patients using the PHQ-9 to identify possible depression and follow up positive screens with further evaluation including current suicidal ideation, prior attempts, mental illness, prior psychiatric hospitalizations, recent biopsychosocial stressors, and availability of firearms. ${ }^{7}$ At present, the USPSTF does not extend these recommendations to adolescents, adults, and older adults, based upon insufficient evidence. ${ }^{8}$ Nonetheless, veteran-specific guidelines could be extended to primary care practices in rural areas and among other populations with a high proportion of firearm ownership or a risk profile similar to veterans. Although identifying the most at-risk patients has proven challenging, screening for depression with follow-up of positive screens is now standard in many primary care practices in the US. Adding inquiry about access to means (i.e., firearms) could potentially be included, reserving more detailed firearm safety and storage counseling interventions for individuals deemed at intermediate or high acute risk of suicide.

The evidence base for counseling on firearm safety and storage counseling is currently inadequate, but at least one recent study reported a reduction in suicide associated with lethal means assessment. ${ }^{5}$ Furthermore, due to the strength of the evidence linking firearm access to increases in suicide rates $^{9,10}$, encouraging actionable steps to remove firearm access among those most at risk is a reasonable approach. In any event, more high-quality studies are needed to corroborate the existing evidence base and to learn more about 
communication frameworks and interventions that can be feasibly implemented and sustained. Because suicide is a relatively rare event, many of these studies will need to be multi-centered and/or conducted in large, integrated health systems.

Even if additional rigorous evidence is available, adding another "unfunded mandate" into outpatient encounters faces an uphill battle. Primary care physicians are already overwhelmed with expectations to conduct and document preventive counseling; adding yet another expectation is viewed with suspicion, if not outright hostility. New coding and documentation guidelines for outpatient Evaluation and Management services that were implemented in 2021 are intended to reduce documentation burden and, in turn, provide more time for counseling and educating patients and caregivers.

To address these concerns, it may be important to incorporate remuneration for physicians providing these services for the most complex patients. For example, to address the issue in the high-risk subpopulation of older adults, firearm safety and storage counseling could be integrated into existing requirements in Medicare for the Annual Wellness Visit (AWV), which is available to all Medicare Part B beneficiaries. In itself a relatively new innovation, billing for the AWV tripled from $8 \%$ of eligible beneficiaries in 2011 to approximately $23 \%$ in 2016. ${ }^{11}$ Adding firearm assessment and safety planning as a voluntary component of the AWV allows it to be billed under the preventive services modifier. This would permit appropriate payment over and above the current rate for an AWV and reward physicians already providing the service.

The actual content of conversations with patients could draw from USPSTF recommendations for veterans as well as existing policy and public health approaches to restrict firearms from those demonstrating risky behavior. For individuals who present an acute risk, consistent with USPSTF recommendations for veterans, absolute restriction from lethal means and observation on a secure unit is usually the best course of action. ${ }^{7}$ In these cases, providers can counsel caregivers to initiate an Extreme Risk Protection Order (ERPO) - a civil order to restrict firearm access - which has been shown to reduce firearm suicide at both the individual level and population level. ${ }^{12}$ Physicians can currently file ERPOs in three states to restrict firearm access, although few have done so. Additional tools for counseling when someone screens as being at intermediate or acute risk of suicide include the mapping of safe storage locations such as has been done in Washington State via the Washington Firearm Safe Storage Map (https://hiprc.org/firearm/firearm-storage-wa/). Similar to Advanced Care Planning, which is an add-on service to the AWV, the sensitive nature of these conversations will usually require more time and effort than discussing less potentially contentious items such as fall prevention or nutrition.

Pursuing a variety of mechanisms to enable and encourage clinicians to regularly include firearm safety and storage counseling in their practices will be required. Evidence- based implementation strategies to integrate these practices into workflow along with provider education will be necessary at the organizational level. Integrating wide-scale firearm safety and storage planning in a program such as Medicare would reinforce that suicide prevention is a national priority and identify a commitment to firearm safety and storage counseling as an important mechanism to help achieve this goal. Additionally, it offers the potential for a records-based, standardized tool for organizations to incorporate into quality improvement programs to reduce firearm suicide and establish a stronger basis of efficacy.

Accurately predicting who will succumb to suicide has been challenging, and widely scalable interventions have yet to demonstrate effectiveness. ${ }^{13}$ Global screening, integrating counseling on lethal means for the most at-risk individuals, and allowing reimbursement for extended counseling are sensible, initial, systems-level approaches with the potential to make a measurable difference.

Corresponding Author: Laura C. Prater, PhD, MPH, MHA; Harborview Injury Prevention and Research Center, Harborview Medical Center, University of Washington, 401 Broadway Suite 2075, Seattle, WA 98104, USA (e-mail: lprater@uw.edu).

\section{Declarations:}

Conflict of Interest: The authors declare that they do not have a conflict of interest.

\section{REFERENCES}

1. Centers for Disease Control. Web-Based injury statistics query and reporting system (WISGARS). US Centers for Disease Control and Prevention.

2. Shenassa ED, Catlin SN, Buka SL. Lethality of firearms relative to other suicide methods: a population based study. J Epidemiol Community Health 2003;57(2): 120-4.

3. Lyons VH, Haviland MJ, Azrael D, Adhia A, Bellenger MA, Ellyson A Rowhani-Rahbar A, Rivara FP. Firearm purchasing and storage during the COVID-19 pandemic. Injury Prevent 2021;27(1):87-92.

4. Ahmedani BK, Simon GE, Stewart C, Beck A, Waitzfelder BE, Rossom R, Lynch F, Owen-Smith A, Hunkeler EM, Whiteside U, Operskalski BH. Health care contacts in the year before suicide death. J Gen Intern Med 2014;29(6):870-7.

5. Boggs JM, Beck A, Ritzwoller DP, Battaglia C, Anderson HD, Lindrooth RC. A quasi-experimental analysis of lethal means assessment and risk for subsequent suicide attempts and deaths. J Gen Intern Med 2020 10:1-6.

6. Butkus R, Doherty R, Bornstein ss. Health and Public Policy Committee of the American College of Physicians. Reducing firearm injuries and deaths in the United States: a position paper from the American College of Physicians. Ann Intern Med 2018;169(10):704-7.

7. Sall J, Brenner L, Millikan Bell AM, Colston MJ. Assessment and management of patients at risk for suicide: synopsis of the 2019 US Department of Veterans Affairs and US Department of Defense clinical practice guidelines. Ann Intern Med 2019;171(5):343-53.

8. O'Connor E, Gaynes BN, Burda BU, Soh C, Whitlock EP. Screening for and treatment of suicide risk relevant to primary care: a systematic review for the US Preventive Services Task Force. Ann Intern Med 2013;158(10):741-54.

9. Kellermann AL, Rivara FP, Somes G, Reay DT, Francisco J, Banton JG, Prodzinski J, Fligner C, Hackman BB. Suicide in the home in relation to gun ownership. N Engl J Med 1992;327(7):467-72. 
10. Studdert DM, Zhang Y, Swanson SA, Prince L, Rodden JA, Holsinger EE, Spittal MJ, Wintemute GJ, Miller M. Handgun ownership and suicide in California. N Engl J Med 2020;382(23):2220-9.

11. Lind KE, Hildreth KL, Perraillon MC. Persistent Disparities in Medicare's Annual Wellness Visit Utilization. Med Care 2019;57(12):984-9.

12. Swanson JW, Easter MM, Alanis-Hirsch K, Belden CM, Norko MA Robertson AG, Frisman LK, Lin HJ, Swartz MS, Parker GF. Criminal justice and suicide outcomes with Indiana's risk-based gun seizure law. 2019 .
13. Perlis RH, Fihn SD. Hard Truths About Suicide Prevention. JAMA Netw Open. 2020;3(10):e2022713-

Publisher's Note: Springer Nature remains neutral with regard to jurisdictional claims in published maps and institutional affiliations. 